\title{
The progress and development of antidepressants
}

\author{
Keyang $\mathrm{Li}^{1, \mathrm{a},{ }^{*}, \dagger}$, and Shiqi Diao ${ }^{2, \mathrm{~b}, *, \dagger}$ \\ ${ }^{1}$ School of Biological Engineering, Nanjing Tech University, Nanjing, Jiangsu 211816 (post), China \\ ${ }^{2}$ School of Department of cell biology, University of Alberta, Edmonton, Alberta AB T6G 2R3 (post), Canada \\ ${ }^{\dagger}$ These authors contributed equally.
}

\begin{abstract}
Major depressive disorder is a common emotional mood disorder disease, which mainly manifests as depression, cognitive impairment, weakened volition activities, loss of appetite, and sleep disorders. Depression is a common mental disorder affecting more than 264 million people worldwide. There are many antidepressant drugs in clinic, e.g., tricyclic antidepressants, monoamine oxidase inhibitors, selective serotonin (5-HT) reuptake inhibitors and selective norepinephrine (NA) reuptake inhibitors. Although great progress has been made in the research of new antidepressant drugs, the current clinical use of drugs still has many shortcomings, e.g., the unmet efficiency, slow onset of action, and multiple adverse reactions, which are difficult to meet the requirements of the increasing number of the depressed patients. This article mainly focuses on the development process of antidepressant drugs and compares their effects. Specifically, the N-methyl-D-aspartate receptor as a new antidepressant has a good effect, which is worthy of further study. These results shed light for providing a research idea for the study of new antidepressants.
\end{abstract}

\section{Introduction}

Major depression is a ubiquitous, toxic, and highly prevalent early-onset disease. It is the main cause of disability in the World Health Organization (WHO) ranking, which is usually associated with heavy social and economic burdens [1]. Generally, it is believed that the high prevalence of depression, various causes, young age of onset, and different treatment responses are the main causes of disability [2]. According to WHO estimations, more than 300 million people worldwide are struggling with depression [3].

Antidepressant therapy is one of the most effective ways to relieve or cure the depressive symptoms of patients with major depression (MDD) [3]. The exploration and clinical application of medicinal antidepressants appeared in the middle of the 20th century. The commonly used clinical antidepressants are the firstgeneration antidepressants derived from the monoamine hypothesis in the 1950s, mainly including tricyclic antidepressants and monoamine oxidase inhibitors [4]. The second-generation antidepressants developed in the late 1980s and early 1990s are generally regarded as a new generation of drugs for the clinical treatment of MDD, represented by selective serotonin reuptake inhibitors (SSRIs) and serotonin-norepinephrine (SNRIs) [5]. However, several monoamine-based drugs have a very low response rate. Plenty of studies have studied the glutamatergic system as a viable target for the treatment of MDD [6]. The non-competitive N-methyl-D-aspartate (NMDA) receptor antagonist ketamine has been producing rapid and sustained antidepressant effects in patients with MDD in a number of clinical studies [6].

\section{Monoamine Hypothesis and Monoamine Based Antidepressants}

The monoamine hypothesis of depression proposes that the basis of depression is the depletion of serotonin, norepinephrine and/or dopamine in the central nervous system [7]. Most antidepressants mainly target monoamine neurotransmitters, trying to increase the presence of these monoamine neurotransmitters in the synaptic space to activate postsynaptic receptors. SSRI provides additional support for the monoamine hypothesis. Recent clinical studies prove that the monoamine hypothesis of MDD needs to be modified, not as simple as the depletion of serotonin, norepinephrine, and dopamine that cause MDD [8]. The revised monoamine hypothesis most consistent with this new data is that the monoamine systems are modulating other brain neurobiologic systems, which have a more primary role in depression $[8,9]$. New antidepressant drugs based on varied types of structure, e.g., N-methyl-D-aspartate receptors, ketamine, as well as esketamine, have achieved ideal results.

\section{Monoamine Oxidase Inhibitor (MAOI)}

MAOI is the earliest drug designed to treat depressive behavior. In 1874, chemist Emile Schafer invented phenelzine, and his students synthesized isoniazid. Primarily, isoniazid was used to treat tuberculosis, but it

\footnotetext{
*Corresponding author: ${ }^{\text {a201821127099} @ n j t e c h . e d u . c n, ~}{ }^{\text {bsdiao@ } @ u a l b e r t a . c a}$
} 
was found in the treatment that isoniazid can cause the patient's mood to rise. On this basis, it is used to cure depression. Non-selective MAOI mainly affects monoamine oxidase (MAO), competes with monoamine neurotransmitters, inhibits the activity of monoamine oxidase, and increases the content of monoamine neurotransmitters in the nervous system to achieve the effect of treating depressive behavior. The main representative drugs for this type are phenelzine, isocarboxazid and tranylcypromine. Due to their severe liver toxicity, they have been seldom used clinically. Type A MAOI can selectively inhibit MAO-A, and its inhibitory effect is reversible, i.e., it has less side effects. It has been widely used in clinical practice. The main drugs are moclobemide and tolo. Sandone. However, when these drugs are used in combination with other drugs, they will interact with each other. The latest research reports that the overdose of moclobemide can cause serotonin syndrome [10]. Therefore, caution should be taken when using these drugs.<smiles>O=C(NCCN1CCOCC1)c1ccc(Cl)cc1</smiles>

4-chloro-N-[2-(morpholin-4-yl)ethyl]benzamide<smiles>NNC(=O)c1cccnc1</smiles>

pyridine-3-carbohydrazide

Figure 1. 2D structure of RIMA moclobemide (left) and pyridine-3-carbohydrazide(right).

\section{Tricyclic Antidepressants}

Tricyclic antidepressants were originally modified by Roland Kuhn from chlorpromazine to imipramine and found that it has a good antidepressant effect. Thus, a series of amitriptyline, protriptyline, doxetine and other Tricyclic antidepressants have been developed subsequently. TCAs block the reuptake of NA and 5-HT by the norepinephrine (NA) and serotonergic (5-HT) nerve endings, and increase the concentration of monoamine transmitters in the synaptic cleft. Compared with MAOI drugs, the improvement of depressive symptoms in clinical manifestations is more significant.<smiles>CN(C)CCC=C1c2ccccc2CCc2ccccc21</smiles>

amitriptyline

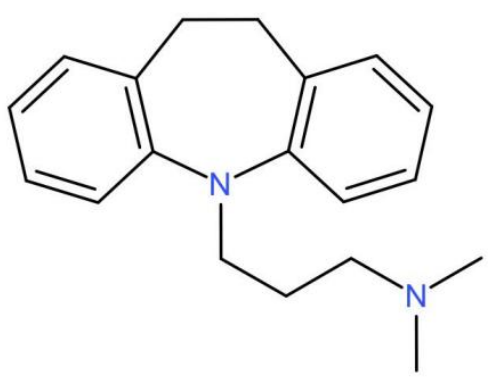

imipramine

Figure 2. 2D structure of amitriptyline(left) and imipramine(right).

\section{Selective serotonin reuptake inhibitors (SSRIs).}

SSRIs were first discovered in the 1980s. Their major mechanism of action is to specifically act on 5-HT transporters, inhibit the reuptake of 5-HT, and thereby increase the content of 5-HT in the synaptic cleft. Literatures have shown that the level of serotonin receptors (5-HTR) is reduced in the brain of patients, and the receptor sensitivity is significantly decreased, indicating that the occurrence of depression is closely related to the number and sensitivity of 5-HTR [11]. Artigas et al. reported that the combination of 5-HT1A receptor partial agonist pindolol and SSRIs can shorten the onset time of SSRIs and enhance the antidepressant effect [12]. Therefore, antidepressants combining SSRIs and 5HT1A have received much attention. SSRIs overcome the shortcomings of MAOI and TCAs, and have the characteristics of antidepressant, strong applicability, and high bioavailability. Especially, they have made great progress in drug safety. Inadvertent overdose of the drug will not cause serious consequences, especially for depression patients who have a suicidal tendency to take the drug. Besides, it has become a first-line antidepressant. The main representative drugs are fluoxetine, paroxetine, fluvoxamine, sertraline and citalopram. The current new drug agomelatine is also effective. It is a melatonin receptor agonist (MT1 and MT2) and has the function of correcting biological circadian rhythm. It is worth noting that fluvoxamine can also specifically bind to the sigma-1 receptor, enhancing the patient's cognitive function, making it superior to other drugs. There are still obvious adverse reactions to SSRIs. Improper use by pregnant women may cause adverse effects on the fetus. 


\section{NMDA receptor antagonists.}

Contemporarily, remarkable progress has been made in the development of new antidepressants based on N-methylDaspartate receptor (NMDAR) targets. As a neurotransmitter, glutamate (Glu) plays an important role in the neuroinformation transmission system. Thereinto, NMDAR is the main subtype of Glu receptor, which is composed of three different subunits: NR1, NR2, and NR3 [13]. In patients with depression, neurons release a large amount of glutamate, and nerve cells have a higher concentration of glutamate. In the environment, NMDAR is over-activated, and a large amount of $\mathrm{Ca}^{2}+$ flows in, which leads to a chain reaction of free radicals. Consequenly, it eventually causes nerve cell death and produces strong excitotoxicity. The currently marketed non-competitive antagonists of NMDAR ion channels are all organic amine compounds, mainly including esketamine, memantine, dizocilpine and lanicemine. On March 5, 2019, the antidepressant drug Spravato with esketamine as the main component was approved by the FDA Approval of listing. Ketamine was once marketed as an anesthetic racemate, but there were adverse reactions such as hallucinations that seriously affected its clinical application [14]. Esketamine is a single dextrorotatory form of ketamine. It can block the NMDAR of inhibitory $\gamma$-aminobutyric acid neurons to achieve de-inhibition of glutamatergic neurons, promote synaptic protein synthesis and brain-derived nutritional factors [15]. Compared with ketamine, esketamine has a higher affinity for NMDAR and fewer adverse reactions. Zhang et al. [16] constructed a dexamethasone depression model and found that (S)ketamine and (R)-ketamine have antidepressant effects, but (R)-ketamine has a longer antidepressant effect than (S)-ketamine. This study is the first to compare the antidepressant effects of (S)-ketamine and (R)-ketamine.
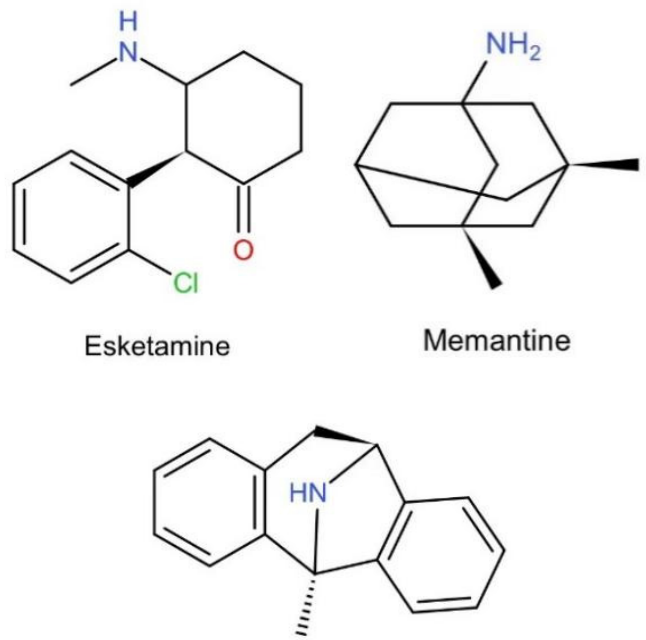

Dizocilpine<smiles>N[C@@H](Cc1ccccn1)c1ccccc1</smiles>

Lanicemine

Figure 3. 2D structure of esketamine, memantine, dizocilpine and lanicemine.

\section{Conclusion}

Depression is a disease of the mental system with complex and diverse pathogenesis. Its typical characteristics are high morbidity, mortality and disability, which seriously threaten the physical and mental health of the human being. In recent years, although people have revealed the underlying mechanism of depression in many aspects, there is still no unified standard. Antidepressants marketed for various pathogenesis have their advantages and disadvantages. The new antidepressant ketamine performs well. Nevertheless, the extent to which the function of TRD patients affects the symptoms and PROs before treatment is unclear. Moreover, the long-term effect needs clinical observation. These results offer a guideline for the research of new antidepressants of N-methyl-D-aspartate receptor and the cause of depression in the future.

\section{References}

1. American Psychiatric Association. Diagnostic and statistical manual of mental disorders. 5th. Arlington, VA: American Psychiatric Publishing; 2013.

2. Kessler, R. C., Berglund, P., Demler, O., Jin, R., Koretz, D., Merikangas, K. R., Rush, A. J., Walters, E. E., Wang, P. S., \& National Comorbidity Survey Replication (2003). The epidemiology of major depressive disorder: results from the National Comorbidity Survey Replication (NCS-R). JAMA, 289(23), 3095-3105. https://doi.org/10.1001/jama.289.23.3095

3. WHO Depression data fact sheet. Geneva: World Health Organization; (2020). Available from: http://www.who.int/mediacentre/factsheets/fs369/en/ (Accessed July 08, 2020)

4. Hirschfeld R. M. (2000). History and evolution of the monoamine hypothesis of depression. The Journal of clinical psychiatry, 61 Suppl 6, 4-6.

5. Lieberman J. History of the use of antidepressants in primary care. J Clin Psychiatry (2003) 5(Suppl 7):610 .

6. Hillhouse, T. M., \& Porter, J. H. (2015). A brief history of the development of antidepressant drugs: from monoamines to glutamate. Experimental and clinical psychopharmacology, 23(1), 1-21. https://doi.org/10.1037/a0038550

7. Delgado PL. Depression: the case for a monoamine 
deficiency. J Clin Psychiatry. 2000;61 Suppl 6:7-11. PMID: 10775018.

8. Heninger, G. R., Delgado, P. L., \& Charney, D. S. (1996). The revised monoamine theory of depression: a modulatory role for monoamines, based on new findings from monoamine depletion experiments in humans. Pharmacopsychiatry, 29(1), 2-11. https://doi.org/10.1055/s-2007-979535

9. FOURNIER J C, DERUBEIS R J, HOLLON S D, et al. Antidepressant drug effects and depression severity: a patient-level meta-analysis [J]. J Am Med Assoc, 2010, 303(1): 47-53.

10. Wu ML, Deng JF. Fatal serotonin toxicity caused by moclobemide and fluoxetine overdose [J]. Chang Gung Med J, 2011, 34(6): 644-649.

11. YATHAM L N, LIDDLE P F, SHIAH IS, et al. Brain serotonin 2 receptors in major depression: a positron emission tomography study [J]. Arch Gen Psychiat, 2000, 57 (9): 850-859.

12. ARTIGAS F, ROMERO L, DE MONTIGNY C, et al. Acceleration of the effect of selected antidepressant drugs in major depression by 5-HT1A antagonists [J]. Trends Neurosci, 1996, 19(9): 378-383

13. XU X, LUO J. Mutations of N-methyl-D-aspartate receptor subunits in epilepsy [J]. Neurosci Bull, 2018, 34(3): 549-565.

14. JAMES W, MURROU G. Ketamine for depression: an update [J]. Biol Psychiat, 2016, 80 (6): 416-418.

15. DUMAN R, AGHAIANIAN G, SANACORA G, et al. Synaptic plasticity and depression: new insights from stress and rapid-acting antidepressants $[\mathrm{J}]$. Nat Med, 2016, 22(3): 238-249.

16. ZHANG JC, LI SX, HASHIMOTO K. R (-) ketamine shows greater potency and longer lasting antidepressant effects than $\mathrm{S}(+)$-ketamine[J]. Pharmacol Biochem Behav, 2014, 116: 137 -141. 